\title{
Heat transfer in plug flow in cylindrical microcapillaries with constant surface heat flux
}

\begin{abstract}
Due to the presence of interfaces, heat transfer can be enhanced by the vortices in liquid plugs in microchannel heat exchangers. The heat transfer in liquid plugs moving in microcapillaries with constant-surface-heat-flux boundary condition is investigated. The effects of the Peclet number and the plug length are studied. Higher Peclet numbers and shorter plug length result in higher Nusselt numbers and lower maximum fluid temperature. However, higher Peclet numbers require higher flow speed, while shorter plug lengths result in higher flow resistance coefficients. The pressure drop needs to be considered in the optimization of microchannel heat exchangers.

Keywords: Plug flow, Vortices, Heat transfer, Heat exchanger, Multiphase microfluidics
\end{abstract}

\section{Nomenclature}

\section{Romans}

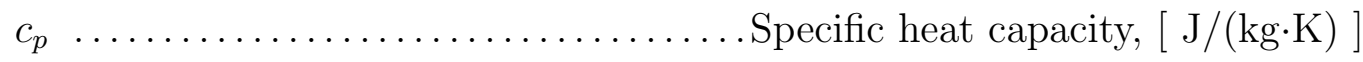
$D_{H} \quad \ldots \ldots \ldots \ldots \ldots \ldots \ldots \ldots \ldots \ldots$ Hydraulic diameter of the channel, $[\mathrm{m}$ ] $h \quad \ldots \ldots \ldots \ldots \ldots \ldots$ Convective heat transfer coefficient, $\left[\mathrm{W} /\left(\mathrm{m}^{2} \cdot \mathrm{K}\right)\right]$ $k \ldots \ldots \ldots \ldots \ldots \ldots \ldots \ldots \ldots \ldots \ldots \ldots$ Thermal conductivity, $[\mathrm{W} /(\mathrm{m} \cdot \mathrm{K})]$

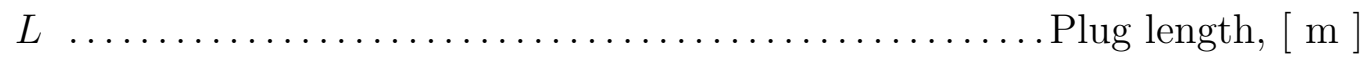




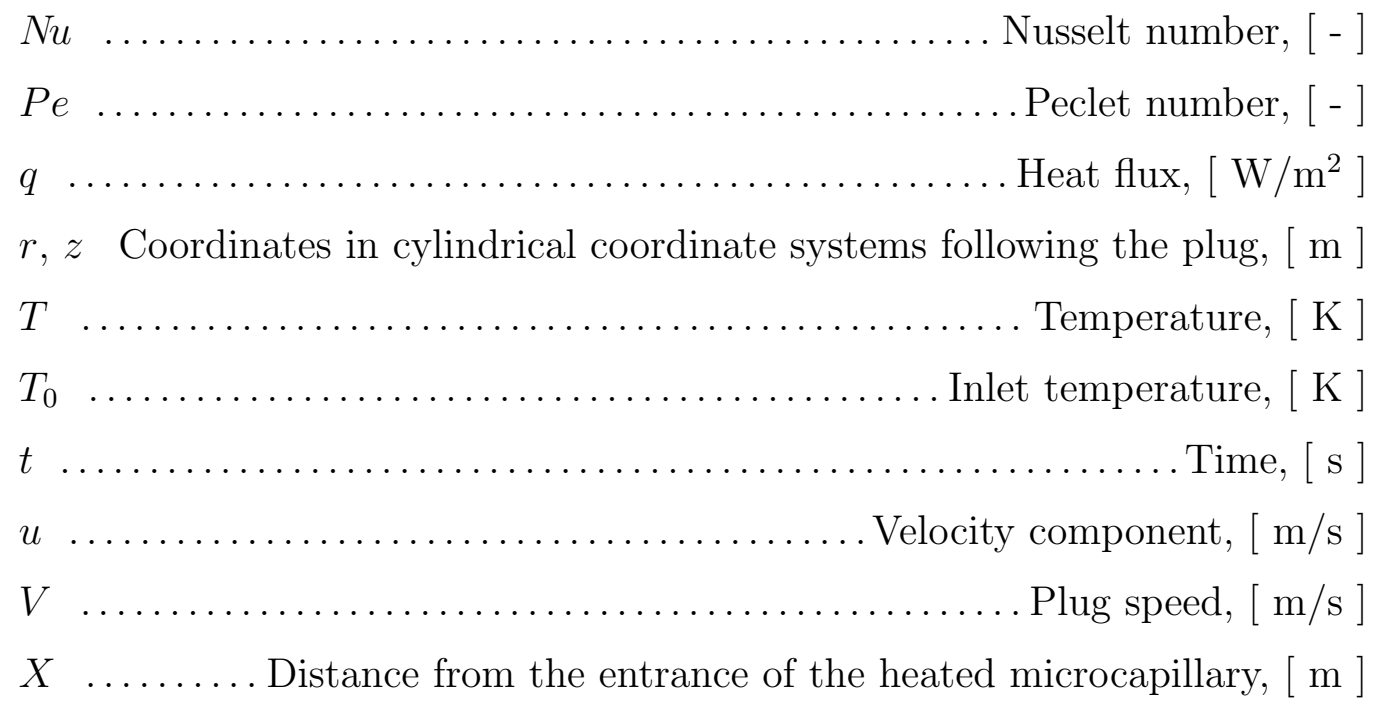

\section{Greeks}

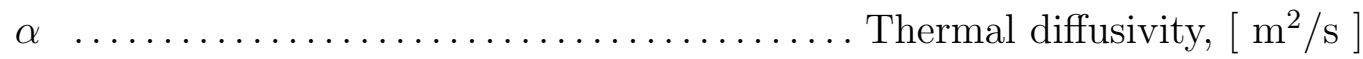

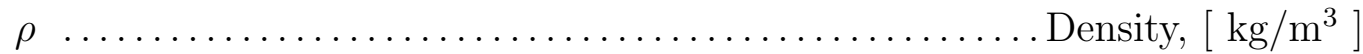

\section{Subscripts}

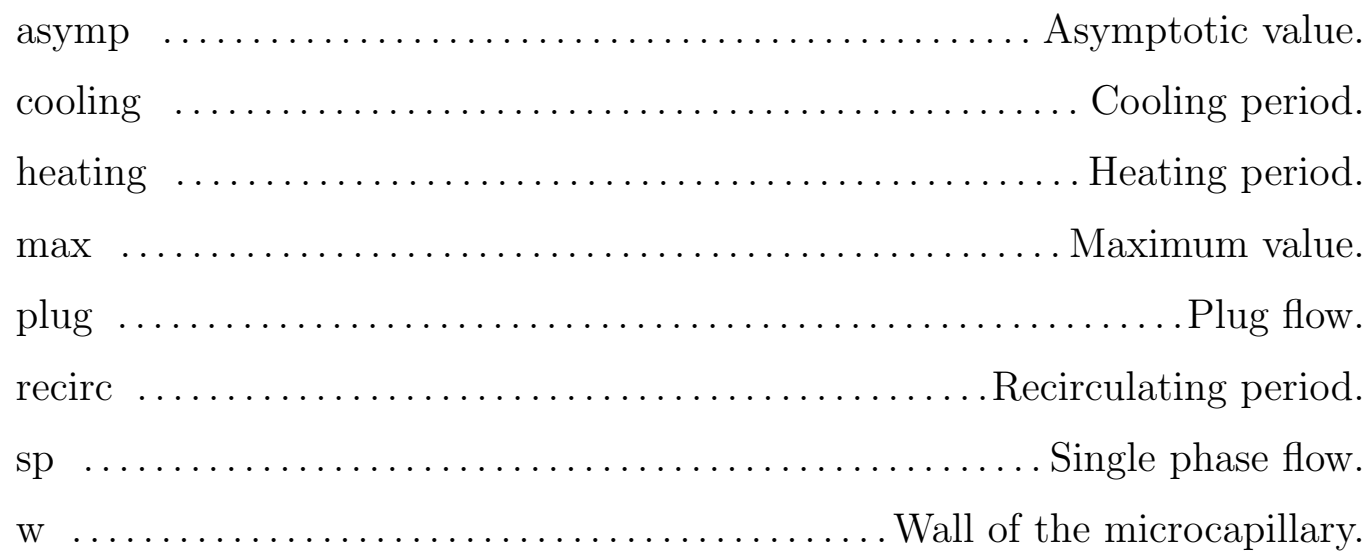

\section{Overscripts}

. Dimensionless variable.

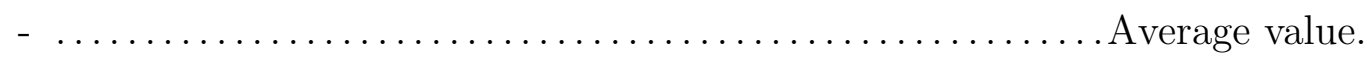

\section{Abbreviations}

$\mu \mathrm{PIV}$

Micro particle image velocimetry. 

. Laser induced fluorescence. TBL . . Thermal boundary layer.

\section{Introduction}

With the high-density packaging and continuing miniaturization of electronic devices, heat generated by electric circuits must be dissipated immediately to prevent failure and to improve reliability. High heat flux ( such as several thousand $\mathrm{W} / \mathrm{cm}^{2}$ ) is expected to be dissipated in high-power electronic devices (Ebadian and Lin, 2011). Many techniques have been developed such as heat pipes (Launay et al., 2007; Vasiliev, 2005), microchannels (Morini, 2004; Palm, 2001), jet impingement (Jambunathan et al., 1992), and spray (Kim, 2007; Lin and Ponnappan, 2003). Microchannels have a lowvolume requirement and can be integrated easily and directly in electronic devices. Another advantage of microchannel heat exchangers is their large surface-volume ratio, which increases its capability in heat dissipation.

In microchannels, due to the small dimension and low fluid speed, the flow is usually laminar and characterized by low Reynolds numbers. Therefore, the heat transfer rate between the fluid and the wall of the microchannel is dominated by thermal diffusion. Without turbulence, the convection of heat is limited. Different strategies have been developed to promote vortices in microchannels, such as curvatures of the microchannels (Liu and Wang, 2009; Naphon and Wongwises, 2006), built-in obstacles in the flow paths (Abbassi et al., 2001; Meis et al., 2010; Turki et al., 2003). The effects of these methods 
are not significant, because most rely on high velocities to achieve secondary flows, whereas high velocity requires an extremely high pressure because the small dimension of the channel produces a high flow resistance.

Vortices can be simply produced by introducing interfaces into the flow in microchannels. For multiphase flow, different flow patterns can be formed, such as bubble/droplet flow, plug flow, annular flow, stratified flow (Kawahara et al., 2002; Triplett et al., 1999). In plug flow (sometimes referred as slug flow, segmented flow, Taylor flow), the liquid droplets adopt plug shapes and occupy almost the entire cross section of the microchannel (Angeli and Gavriilidis, 2008). With the presence of the interface, vortices are formed and dominate the whole liquid plug (Che et al., 2011; Handique and Burns, 2001). With the help of the vortices, heat and mass transfer (Günther et al., 2005; Song et al., 2003a,b) in the liquid plug can be greatly enhanced.

The study of heat transfer in plug flow in microchannels is not as extensive as the single-phase counterpart. A possible reason is that due to the presence of the interface, vortices are formed and the recirculating flow field is more complex than the Poiseuille flow in single-phase flow. Some experimental studies of heat transfer with phase change in microchannel were carried out (Chen and Cheng, 2005; Peng and Wang, 1993; Thome, 2004) and the latent heat of the fluid also enhances the capability of thermal management. However, the unstable flow nature (such as boiling) also increases the complexity of the analyses. Furthermore, in many applications, a stable flow is preferred because it is easier to control than unstable phase change heat transfer.

To quantitatively study the physics of heat transfer enhancement in plug 
flow, it is ideal to measure both the velocity field and the temperature field simultaneously. Micro particle imaging velocimetry ( $\mu$ PIV) is an optical measurement method and it can provide the $2 \mathrm{D}$ or $3 \mathrm{D}$ velocity fields in microchannels, while laser induced fluorescence (LIF) method is a technique to measure the temperature field in microchannels. However, these methods require that the refractive indices of the different phases should be matched to avoid optical distortions at the interface (Kim et al., 2004; Oishi et al., 2009). Walsh et al (2010) measured the surface temperature of a stainless tube using a high resolution infrared thermography system, but did not obtain the temperature field in the liquid plugs.

To analyze the heat transfer process in plug flow, Muzychka et al (2010) proposed a simplified model by assuming a uniform velocity profile throughout the plug and considering the heat transfer process as a Graetz problem with steady state heat transfer. To studies the interplay between the flow field and the temperature field, it is better to considering the recirculating vortices in the liquid plugs. There are numerical methods which are able to predict the interface shape and obtain the velocity field, such as the front tracking method (Muradoglu et al., 2007), the volume of fluid method (Scardovelli and Zaleski, 1999; Taha and Cui, 2004), the level set method (Chen et al., 2009; Osher and Fedkiw, 2001), the Lattice Boltzmann method (Yu et al., 2007; Zhang, 2010), and the moving-grid method (Fujioka and Grotberg, 2004). However, these numerical methods are usually complex and computation-time consuming, and their applications in heat transfer in plug flow are limited.

In the earlier work (Che et al., 2012), we performed numerical simulation 
of heat transfer based on an analytical model of flow field in liquid plugs in a 2D microchannels subjecting to constant-surface-temperature boundary condition. This approach was proven to be convenient and effective to study the heat transfer process in plug flow. The results show that the recirculating vortices significantly affect the temperature contours and the heat transfer process with constant-surface-temperature boundary condition. Here in this paper, we extend this approach to study the heat transfer in plug flow within cylindrical capillaries subjecting to a constant-surfaceheat-flux boundary condition, as shown in Fig. 1. The constant-surfaceheat-flux boundary condition is closely approximated, for example, when the surface is bonded to a heater (or cooler) which supplies (or absorbs) heat at a constant rate. Cylindrical capillaries are commonly used in many applications whereas 2D microchannels correspond to slit microchannels with large aspect ratios. When a liquid plug is moving in a cylindrical capillary, the vortices in the plug are of toroidal shape, which are different from those in $2 \mathrm{D}$ microchannels where they are a pair of counter-rotating vortices. Besides in microchannel heat exchangers, plug flow appears in many biological systems, such as in lungs and tracheas. The flow paths in these biological systems can be approximated as cylindrical capillaries. The heat and mass transfer is accomplished across the fluid and the wall of biological systems, and the studies shows promising application in medical treatments.

The paper is structured as follows. The method to study the flow and the heat transfer is provided in Section 2. The results are discussed in Section 3, and the effects of the Peclet number and the plug length are analyzed. 


\section{Mathematical modeling}

\subsection{Modeling of flow field in liquid plugs}

For plug flow in cylindrical capillaries, the flow field in the plug can be modeled analytically (Che et al., 2011). A cylindrical coordinate is built following the liquid plug and the following dimensionless variable can be used as follows,

$$
\hat{r} \equiv \frac{r}{R}, \quad \hat{z} \equiv \frac{z}{R}, \quad \hat{L} \equiv \frac{L}{R}, \quad \hat{u}_{r} \equiv \frac{u_{r}}{V}, \quad \hat{u}_{z} \equiv \frac{u_{z}}{V} .
$$

The velocity components take the form

$$
\begin{aligned}
& \hat{u}_{r}=-\sum_{n=1}^{\infty} \alpha_{n} \cos \left(\alpha_{n} \hat{z}\right) A_{n} \frac{I_{1}\left(\alpha_{n} \hat{r}\right) I_{2}\left(\alpha_{n}\right)-\hat{r} I_{1}\left(\alpha_{n}\right) I_{2}\left(\alpha_{n} \hat{r}\right)}{I_{2}^{2}\left(\alpha_{n}\right)} \\
& \hat{u}_{z}=\sum_{n=1}^{\infty} \sin \left(\alpha_{n} \hat{z}\right) A_{n} \frac{-\alpha_{n} \hat{r} I_{1}\left(\alpha_{n}\right) I_{1}\left(\alpha_{n} \hat{r}\right)+\alpha_{n} I_{0}\left(\alpha_{n} \hat{r}\right) I_{2}\left(\alpha_{n}\right)}{I_{2}^{2}\left(\alpha_{n}\right)}
\end{aligned}
$$

where

$$
\begin{gathered}
\alpha_{n}=n \pi / \hat{L}, \\
A_{n}=\frac{2\left[(-1)^{n}-1\right]}{F_{n}^{\prime}(1) n \pi}, \\
F_{n}^{\prime}(\hat{r})=\frac{-n \pi \hat{r}^{2} I_{1}\left(\alpha_{n}\right) I_{1}\left(\alpha_{n} \hat{r}\right)+n \pi \hat{r} I_{0}\left(\alpha_{n} \hat{r}\right) I_{2}\left(\alpha_{n}\right)}{\hat{L} I_{2}^{2}\left(\alpha_{n}\right)},
\end{gathered}
$$

where $I_{n}$ is the modified Bessel function of the first kind of order $n$.

\subsection{Analysis of heat transfer}

\subsubsection{Governing equation}

For the heat transfer in liquid plugs in cylindrical capillaries, the governing equations is

$$
\rho\left(\frac{\partial T}{\partial t}+\frac{1}{r} \frac{\partial\left(r u_{r} T\right)}{\partial r}+\frac{\partial\left(u_{z} T\right)}{\partial z}\right)=\frac{k}{c_{p}}\left[\frac{1}{r} \frac{\partial}{\partial r}\left(r \frac{\partial T}{\partial r}\right)+\frac{\partial^{2} T}{\partial z^{2}}\right]
$$


where $\rho, k$, and $c_{p}$ are the density, the thermal conductivity, and the specific heat capacity of the liquid, respectively. By introducing the following dimensionless variables,

$$
\hat{t} \equiv \frac{t}{R / V}, \quad \hat{T} \equiv \frac{T-T_{0}}{q_{\mathrm{w}} R / k}
$$

Eq. (7) can be normalized as

$$
\frac{\partial \hat{T}}{\partial \hat{t}}+\frac{1}{\hat{r}} \frac{\partial\left(\hat{r} \hat{u}_{r} \hat{T}\right)}{\partial \hat{r}}+\frac{\partial\left(\hat{u}_{z} \hat{T}\right)}{\partial \hat{z}}=\frac{1}{P e}\left[\frac{1}{\hat{r}} \frac{\partial}{\partial \hat{r}}\left(\hat{r} \frac{\partial \hat{T}}{\partial \hat{r}}\right)+\frac{\partial^{2} \hat{T}}{\partial z^{2}}\right]
$$

where $P e \equiv \frac{R V}{\alpha}$ is the Peclet number and $\alpha \equiv \frac{\lambda}{\rho c_{p}}$ is the thermal diffusivity. The heat transfer equation (9) is discretized using the finite volume method (Patankar, 1980) on staggered grid. The velocities components $\left(\hat{u}_{r}, \hat{u}_{z}\right)$ are obtained directly from Eqs. (2) and (3).

\subsubsection{Boundary conditions}

When the liquid plug is moving in the capillary, the wall of the capillary is maintained at a constant surface heat flux, as shown in Fig. 1. Therefore, the boundary condition of the heat transfer for the liquid plug is

$$
-k \frac{\partial T}{\partial r}=-q_{\mathrm{w}} \quad \text { at } \quad r=R .
$$

According to Eq. (8), the boundary condition in Eq. (10) can be normalized as

$$
\frac{\partial \hat{T}}{\partial \hat{r}}=1 \quad \text { at } \quad \hat{r}=1
$$

Along the axis of the cylindrical capillary, the boundary condition for heat transfer in the plug is

$$
\frac{\partial T}{\partial r}=0 \quad \text { at } \quad r=0
$$




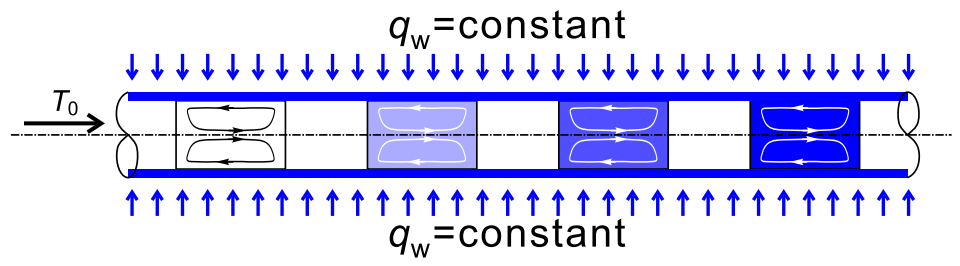

Figure 1: Schematic diagram of heat transfer for plugs in a cylindrical capillary with constant surface heat flux.

and in dimensionless form, it is

$$
\frac{\partial \hat{T}}{\partial \hat{r}}=0 \quad \text { at } \quad \hat{r}=0 .
$$

\subsubsection{Characterization of heat transfer process}

To characterize the heat transfer process of plug flow in the capillary, the Nusselt number $N u$ and the maximum fluid temperature $\hat{T}_{\max }$ are used. For the comparison between the plug flow and the single phase, $N u$ and $\hat{T}_{\max }$ are calculated (i) at an axial location $\hat{X}$ from the entrance of the capillary for the single phase flow, and (ii) at the instant when the plug passes the axial location $\hat{X}$ for the plug flow. According to the dimensionless group in Eqs. (1) and (8), the dimensionless time $\hat{t}$ and the dimensionless axial location of the liquid plug in the capillary $\hat{X}$ is linked as

$$
\hat{t}=\hat{X}
$$

Nusselt number. The Nusselt number characterizes the ratio of convective to conductive heat transfer normal to wall of the capillary. It is defined as

$$
N u \equiv \frac{h D_{H}}{k}
$$


where $D_{H}=2 R$ is the hydrodynamic diameter of the channel, and $h$ is the convective heat transfer coefficient on the wall.

For plug flow, the convective heat transfer coefficient $h_{\text {plug }}$ in the constantsurface-heat-flux boundary condition is defined as

$$
h_{\text {plug }} \equiv \frac{q_{\mathrm{w}}}{\bar{T}_{\mathrm{w}}-\bar{T}_{\mathrm{plug}}}
$$

where $\bar{T}_{\text {plug }}$ is the mean fluid temperature of a plug unit (Incropera and DeWitt, 2002)

$$
\bar{T}_{\text {plug }}(X) \equiv \frac{\int_{A_{\text {plug }}} \rho c_{p} u_{x} T d A_{\text {plug }}}{\int_{A_{\text {plug }}} \rho c_{p} u_{x} d A_{\text {plug }}},
$$

and $\bar{T}_{\mathrm{w}}$ is the mean temperature of the wall over the plug length

$$
\left.\bar{T}_{\mathrm{w}}(X) \equiv \frac{1}{L} \int_{0}^{L} T\right|_{r=R} d z
$$

In dimensionless form, the mean fluid temperature of the plug unit is

$$
\hat{\bar{T}}_{\text {plug }}(\hat{X}) \equiv \frac{\int_{0}^{1} d \hat{y} \int_{0}^{\hat{L}} \hat{u}_{x} \hat{T} d \hat{x}}{\int_{0}^{1} d \hat{y} \int_{0}^{\hat{L}} \hat{u}_{x} d \hat{x}},
$$

and the mean wall temperature over the plug length is

$$
\left.\hat{\bar{T}}_{\mathrm{w}}(\hat{X}) \equiv \frac{1}{\hat{L}} \int_{0}^{\hat{L}} \hat{T}\right|_{\hat{r}=1} d \hat{z}
$$

Therefore, the Nusselt number of plug flow can be obtained as

$$
N u_{\text {plug }}=\frac{2 \hat{L}}{\left(\hat{\bar{T}}_{\mathrm{w}}-\hat{\bar{T}}_{\text {plug }}\right)} .
$$

For single phase flow, the mean fluid temperature $\hat{\bar{T}}_{\mathrm{sp}}(\hat{X})$ refers to the mean temperature of the fluid over the cross section at the axial location 
$\hat{X}$ of the capillary, and in dimensionless form, it is (Incropera and DeWitt, 2002)

$$
\hat{\bar{T}}_{\mathrm{sp}}(\hat{X}) \equiv \frac{\int_{0}^{1} \hat{u}_{x} \hat{T} d \hat{y}}{\int_{0}^{1} \hat{u}_{x} d \hat{y}} .
$$

Consequently, for single phase flow in microchannel, the convective heat transfer coefficient is

$$
h_{\mathrm{sp}} \equiv \frac{q_{\mathrm{w}}}{T_{\mathrm{w}}-T_{\mathrm{sp}}} .
$$

And the Nusselt number is

$$
N u_{\mathrm{sp}}=\frac{2}{\hat{T}_{\mathrm{w}}-\hat{\bar{T}}_{\mathrm{sp}}} .
$$

Maximum fluid temperature. With a constant-surface-heat-flux boundary condition maintained on the wall, a favorable situation for heat exchangers is that the fluid remains at a reasonable low temperature in electronic devices. The highest tolerable fluid temperature is usually a requirement when designing heat exchangers. High temperature may damage the devices. In biological systems, high temperature can cause irreversible problems such as cell death. With this concept, a maximum fluid temperature can be defined in dimensionless form as

$$
\hat{T}_{\max } \equiv \max (\hat{T}) .
$$

For single phase flow, $\hat{T}_{\max }$ is searched across the cross section at the axial location $\hat{X}$ of the microchannel, while for plug flow, $\hat{T}_{\max }$ is searched throughout the plug at the instant when the plug passes the cross section, i.e., $\hat{t}=\hat{X}$. 


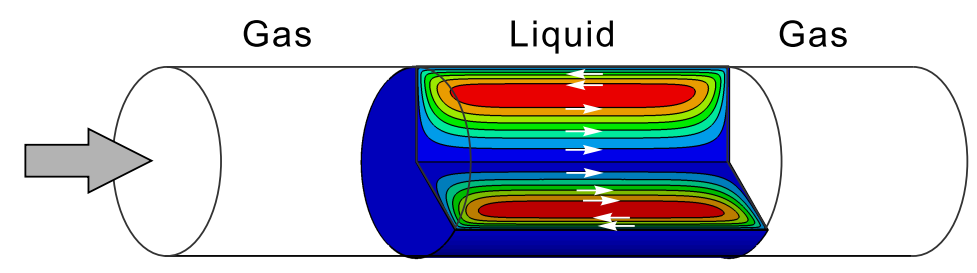

Figure 2: Flow field in a typical liquid plug in cylindrical microchannel. The dimensionless plug length is $\hat{L}=4$.

\section{Results and discussion}

\subsection{Flow field in a typical liquid plug}

Based on the analytical expression shown in Eqs. (2) and (3), the flow field in a typical liquid plug in a capillary is shown in Figure 2. The flow is axisymmetrical with respect to the axis of the channel. When the liquid in the plug is moving forward near the axis of the capillary, it is blocked by the front interface and moves radially toward the wall of the capillary. With a moving reference following the plug, the liquid near the wall moves backward, and it is blocked by the rear interface and thereafter moves convergingly toward the axis of the capillary. Consequently, recirculating flow with a toroidal shape is formed in the plug due to the presence of the front/rear interfaces. The recirculating flow can effectively enhance the heat transfer between the wall and the liquid by advecting the liquid in the plug.

\subsection{Intermittent heating and cooling in liquid plugs}

When fluid particles are recirculating in the liquid plugs, they experience intermittent heating and cooling in the plugs. When the fluid particles are in the vicinity of the wall of the capillary, they receive heat from the wall, while when they are further away from the wall, they reject heat to the surrounding 


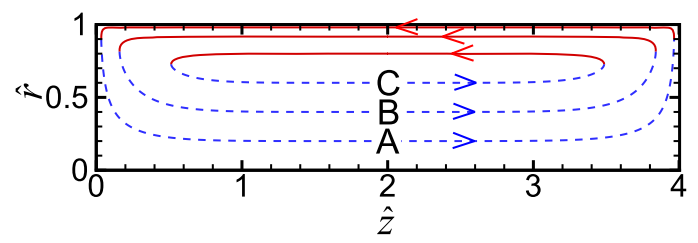

(a)

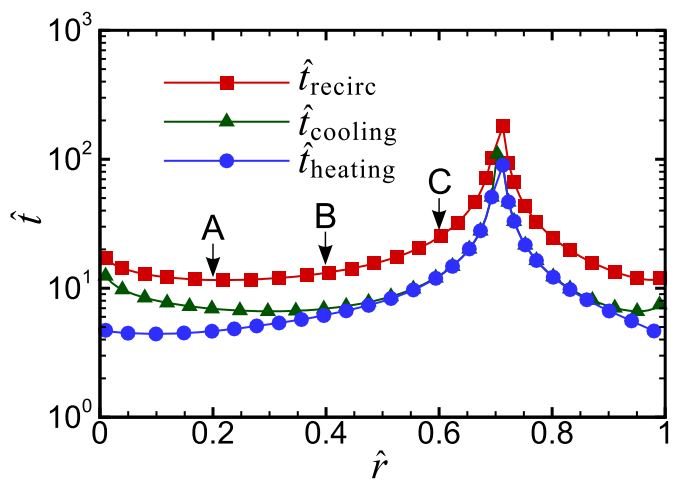

(b)

Figure 3: (a) Fluid particles, when flowing along the streamlines, experience intermittent heating and cooling in the plugs. The solid segments indicate the heating periods, while the dashed segments indicate the cooling periods. (b) Variation of the recirculating period $\hat{t}_{\text {recirc }}$, heating period $\hat{t}_{\text {heating }}$, and cooling period $\hat{t}_{\text {cooling }}$ with the radial position of the fluid particle. The dimensionless plug length is $\hat{L}=4$.

fluid, as show in Figure 3. The recirculating period $t_{\text {recirc }}$ of a fluid particle refers to the period during which the fluid particle flows in the liquid plug for one cycle and gets back to its original position. The recirculation time of fluid particles can be divided into the heating period $\hat{t}_{\text {heating }}$ and the cooling period $\hat{t}_{\text {cooling }}$, as shown in Figure 3 a. In a liquid plug, $t_{\text {recirc }}$ are identical for all the fluid particle along the same streamline, but varies for particles on different streamlines, as shown in Figure 3b. 


\subsection{Stages of heat transfer in a typical liquid plug}

The time evolution of the temperature distribution in a liquid plug moving through a capillary is shown in Figure 4a, and their corresponding points are marked in Figure 4b. The channel wall is subjected to a constant heat flux, the dimensionless plug length is $\hat{L}=4$, and the Peclet number is $P e=100$. The variation of $N u$ [Eq. (21)], $\hat{\bar{T}}_{\mathrm{w}}$ [Eq. (20)], and $\hat{\bar{T}}_{\text {plug }}$ [Eq. (19)] are plotted against the axial location $\hat{X}$ in Figure 4 b. The heat transfer in plug flow can be divided into three subsequent stages: (i) development of thermal boundary layer; (ii) advection of heated/fresh fluid in the plug; and (iii) thermally fully developed flow.

Stage I: Development of thermal boundary layer (TBL), [Figure 4, (Instants B-C)]. When the plug contacts the heated wall, a thin liquid layer in the immediate vicinity of the wall rapidly increases its temperature and forms a TBL, while the fluid outside the TBL is unaffected [Figure 4a (Instant B)]. Due to the negative flow direction in the TBL along the wall, the TBL is thinner at the front of the plug, while it is thicker near the rear. The thickness of the TBL grows with time until the TBL reaches the centreline of the channel [Figure 4a (Instant C)], which marks the end of Stage I and the beginning of Stage II.

The Nusselt number decreases as the TBL develops. Due the constantsurface-heat-flux boundary condition, a constant temperature gradient is maintained at the wall. The TBL is thin initially [Figure 4a (Instant B)], which results in a small temperature difference between the wall and the plug $\left(\hat{\bar{T}}_{\mathrm{w}}-\hat{\bar{T}}_{\text {plug }}\right)$ and a large $N u$ [Figure $\left.4 \mathrm{~b}\right]$. However, as the TBL develops, $N u$

decreases rapidly, and $\left(\hat{\bar{T}}_{\mathrm{w}}-\hat{\bar{T}}_{\text {plug }}\right)$ increases correspondingly with increasing 
$\hat{X}$, [Figure $4 \mathrm{~b}]$.

Stage II: Advection of heated/fresh fluid in the plug, [Figure 4, (Instants D-G)]: Due to the presence of the front interface, the fresh fluid is being transported from the central region of the plug to the heated wall; while due to the presence of the rear interface, the heated fluid is being transported from the wall towards the central region. The advection of heated/fresh fluid reduces the temperature difference $\left(\hat{\bar{T}}_{\mathrm{w}}-\hat{\bar{T}}_{\text {plug }}\right)$. At Stage II, the advection of the heated/fresh fluid results in the oscillation of the Nusselt number, as shown in Figure 4b.

When the fresh fluid in the central region of the plug is being transported to the heated wall by the recirculating flow [Figure 4 (Instants D-E)], $\hat{\bar{T}}_{\mathrm{w}}$ remains almost constant, while $\hat{\bar{T}}_{\text {plug }}$ increases linearly. In this period, $\left(\hat{\bar{T}}_{\mathrm{w}}-\right.$ $\hat{\bar{T}}_{\text {plug }}$ ) decreases with increasing $\hat{X}$, hence, the Nusselt number [according to Eq. (21)] increases. As the heated fluid in the plug is transported back to the heated wall [Figure 4 (Instant F)], $\hat{\bar{T}}_{\mathrm{w}}$ and $\left(\hat{\bar{T}}_{\mathrm{w}}-\hat{\bar{T}}_{\text {plug }}\right)$ increase, which results in a decrease in $N u$. The periodic oscillation of $N u$ is damped as the temperature difference $\left(\hat{\bar{T}}_{\mathrm{w}}-\hat{\bar{T}}_{\text {plug }}\right)$ gradually approaches a constant value.

Stage III: Thermally fully developed flow, [Figure 4, (Instants H-I)]: Heat transfer continues as a constant heat flux is imposed at the wall, $\hat{\bar{T}}_{\text {plug }}$ increases as the plugs move in the capillary. At this fully developed stage, the temperature difference between the plug and the wall $\left(\hat{\bar{T}}_{\mathrm{w}}-\hat{\bar{T}}_{\text {plug }}\right)$ remains constant with increasing $\hat{X}$. As shown in Figure 4a (Instants H-I), the shapes of the temperature contours remain unchanged. 


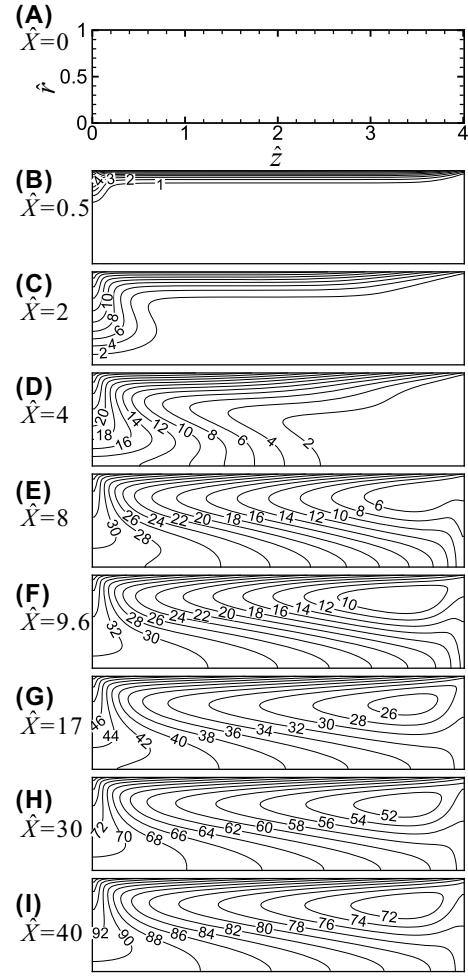

(a)

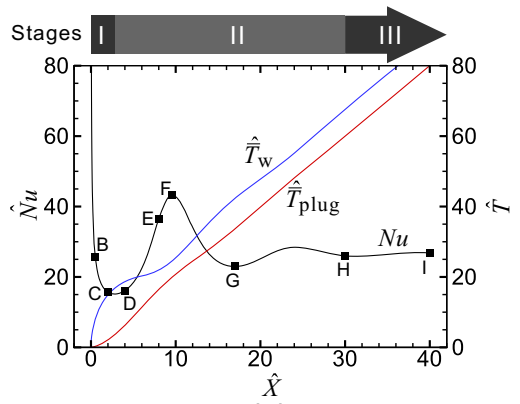

(b)

Figure 4: Three stages of the heat transfer process in a typical liquid plug $(\hat{L}=4, P e=$ 100): Stage I (B-C): development of thermal boundary layer; Stage II (D-F): advection of the heated/fresh fluid in the plug; and Stage III $(\mathrm{G}-\mathrm{H})$ : thermally fully developed flow. (a) Time evolution of temperature distribution in the liquid plug. (b) Variation of the Nusselt number $N u$, the mean fluid temperature of the plug unit $\hat{\bar{T}}_{\text {plug }}$ and the mean temperature of the wall over the plug length $\hat{\bar{T}}_{w}$. The marked points correspond to the instants in (a). 


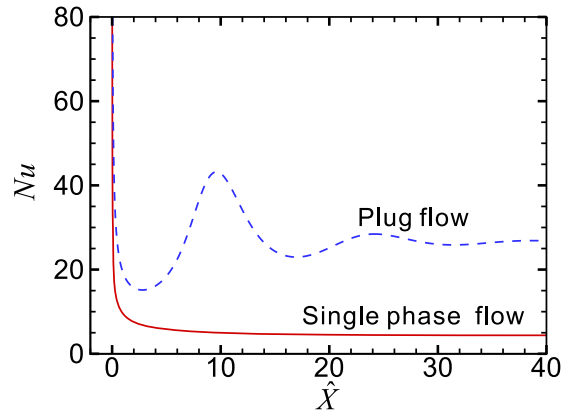

(a)

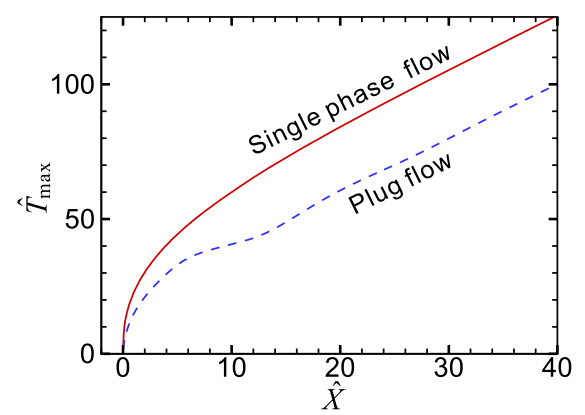

(b)

Figure 5: Comparison between the plug flow with single phase in capillaries $(\hat{L}=4$, $P e=100$ ). (a) Evolution of $N u$ for plug flow and for single phase flow. (b) Maximum fluid temperature $\hat{T}_{\max }$ for plug flow and for single phase flow.

\subsection{Comparison with single phase flow}

To compare between the plug flow and the single phase flow, the Nusselt number $N u$ and the maximum fluid temperature $\hat{T}_{\max }$ are plotted in Figures $5 \mathrm{a}$ and $\mathrm{b}$.

For single phase flow, the Nusselt number is large near the entrance of the channel and decays along the microchannel to the asymptotic limit $\left(N u_{\text {asymp,sp }}=4.4\right)$ due to the development of the thermal boundary layer along the wall, as shown in Figure 5a. In contrast, as the liquid plug is moving in the capillary, the Nusselt number oscillates during Stage II, and achieve a asymptotic limit of Nusselt number much larger than the single phase flow.

The maximum fluid temperature is much lower for plug flow than single phase flow, as shown in Figure 5b. This is because the recirculating flow in liquid plugs effectively homogenizes the temperature field in the liquid plug, and consequently reduces the maximum fluid temperature. 


\subsection{Effect of Peclet number}

With a constant surface heat flux on a wall, $N u$ and $\hat{T}_{\max }$ versus $\hat{X}$ for different Peclet numbers are plotted in Figure 6. The plug length is fixed at 4 while $P e$ is varied from 4 to 1024. At a low Peclet number $(P e=4)$, the Nusselt number is relatively low, with an asymptotic limit of $N u_{\text {asymp, } P e=4}=$ 7.4 at Stage III. The Nusselt number increases with increasing $P e$. At a high Peclet number $(P e=1024)$, the asymptotic limit of $N u$ at Stage III increases to $N u_{\text {asymp }, P e=1024}=30.9$. The increase in $N u$ with increasing $P e$ is mainly due to the increase in the advection effect, which effectively transports the heated fluid from the wall to the central region of the plug and transports the fresh fluid from the central region to the heated wall.

The influence of $P e$ on $\hat{T}_{\max }$ versus the axial distance $\hat{X}$ [Figure $6 \mathrm{~b}$ ] shows that $\hat{T}_{\text {max }}$ decreases with increasing $P e$. This is because a fast flow (high $P e$ ) has a short residence time in the heated channel. To further quantitatively compare the effect of $P e$ between the single phase flow and the plug flow, $\hat{T}_{\max }$ is plotted against $P e$ in Figure 7 . At a low Peclet number $(P e=4)$, the difference in $\hat{T}_{\max }$ between the single phase flow and the plug flow is

insignificant. $\hat{T}_{\max }$ decreases as $P e$ increases for both single phase flow and plug flow. At a high Peclet number, $\hat{T}_{\max }$ for single phase flow is higher than that for plug flow. When $P e=1024, \hat{T}_{\max }$ for single phase flow is 2.67 times for plug flow. These results indicates that at a high $P e$, plug flow can significantly reduce $\hat{T}_{\max }$ by the recirculating flow within the liquid plugs.

In the design and optimization of microchannel heat exchangers, a higher Peclet number is preferred in order to obtain a lower maximum fluid temperature. However, high Peclet number requires a higher flow 


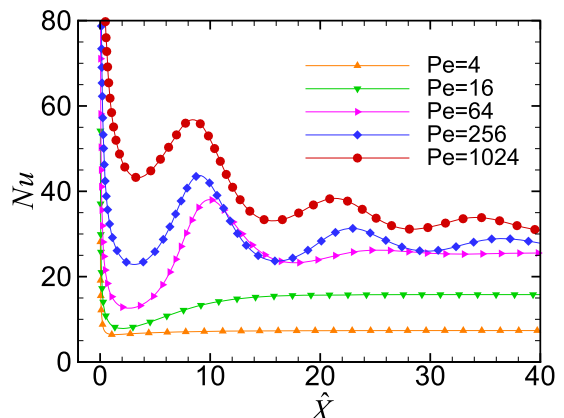

(a)

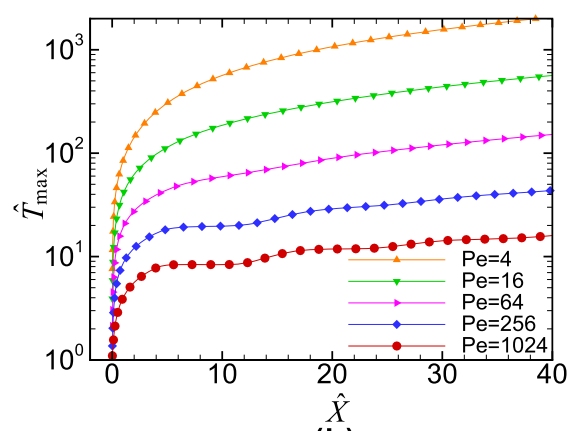

(b)

Figure 6: Effect of Peclet number on heat transfer in plug flow in a capillary $(\hat{L}=4)$. (a) Nusselt number $N u$; (b) Maximum temperature $\hat{T}_{\max }$.

speed, which increases the pressure drop along the microchannel and the power consumption. Therefore, the compromising between the pressure drop and the maximum fluid temperature should be considered in selecting an optimum Peclet number.

\subsection{Effect of plug length}

For heat transfer in plug flow with the constant-surface-heat-flux boundary condition, the effect of plug length on $N u$ is shown in Figure 8a. The Peclet number is fixed at 100 and the dimensionless plug length is varied from 1 to 8. For longer plugs, the periods of Stage I and Stage II are longer than those of short plugs. This is because the recirculating period is longer for longer plugs as shown in Figure 10. In addition, the oscillation of $N u$ is weaker for longer plugs than that for short plugs. This is because for long plugs, heat have dissipated to the surrounding fluid before the heated fluid is transported back to the wall.

The effect of the plug length on the maximum fluid temperature for constant surface heat flux is shown in Figure 8b. Due to the initial oscillation 


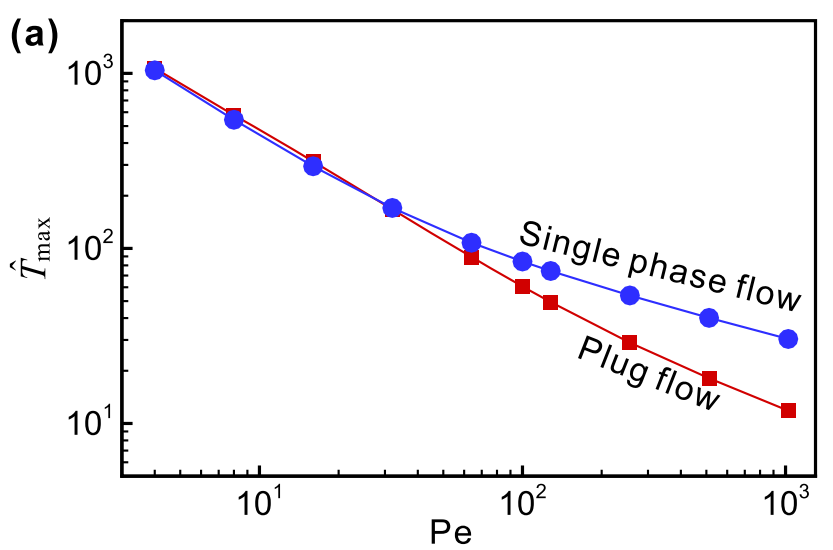

(b) $\mathrm{Pe}=4$

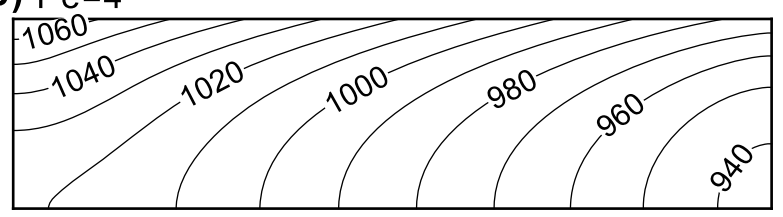

(c) $\mathrm{Pe}=64$

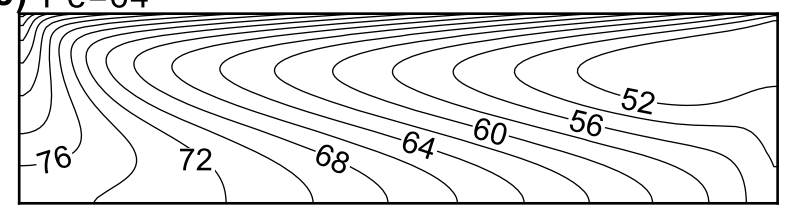

(d) $\mathrm{Pe}=1024$

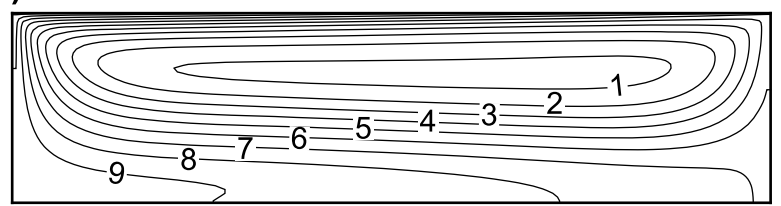

Figure 7: Maximum fluid temperature $\hat{T}_{\max }$ at $\hat{X}=20$ at different Peclet numbers. (a) Variation of $\hat{T}_{\max }$ with $P e$; (b)-(d) Temperature contours at different Peclet numbers $(P e=4,64,1024)$. 
of $N u$, the increasing rate of the maximum fluid temperature $d \hat{T}_{\max } / d \hat{X}$ varies with time. As $N u$ approaches its asymptotic limit, $d \hat{T}_{\max } / d \hat{X}$ remains constant, which reflects that the heat transfer gradually becomes fully developed (Stage III). From the maximum fluid temperature in Figure 8b, we can also find that $\hat{T}_{\max }$ in short plugs is lower than that in long plugs. This is due to the recirculating period $\hat{t}_{\text {recirc }}$ for fluid particles in plugs of different lengths [Figure 10]. A longer plug results in a longer recirculating time $\hat{t}_{\text {recirc }}$, a longer heating period $\hat{t}_{\text {heating, }}$, and a longer cooling period $\hat{t}_{\text {cooling, }}$ and vice versa. A longer $\hat{t}_{\text {heating }}$ results in a higher $\hat{T}_{\max }$, since $\hat{T}_{\max }$ is the maximum fluid temperature that fluid particles can achieve during the heating period near the heated wall.

Short plugs are preferred than long plugs to obtain lower maximum fluid temperature and higher Nusselt numbers. However, the flow resistance coefficient in short plugs is also higher than that in longer plugs (Che et al., 2011), which increases the pressure drop along the microchannel. Therefore, during the design and optimization of microchannel heat exchangers, the compromising between the pressure drop and the maximum fluid temperature should be considered in selecting an optimum plug length.

\section{Conclusions}

In this paper, the heat transfer of liquid plugs moving in capillaries with constant-surface-heat-flux boundary condition is investigated systematically. By incorporating the analytical flow field, the heat transfer process in liquid plug moving in micapillaries are simulated using finite volume method. The effects of Peclet number and the plug length are studied. Three stages of 


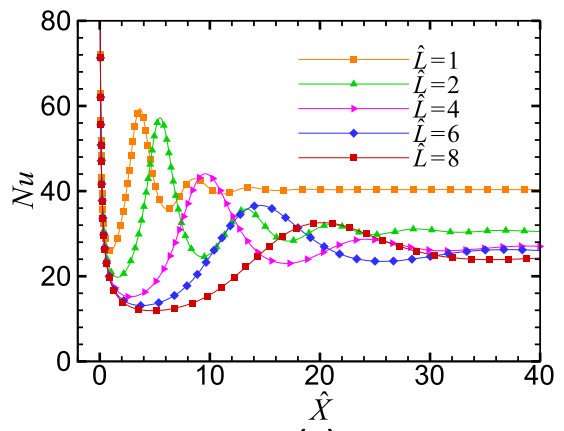

(a)

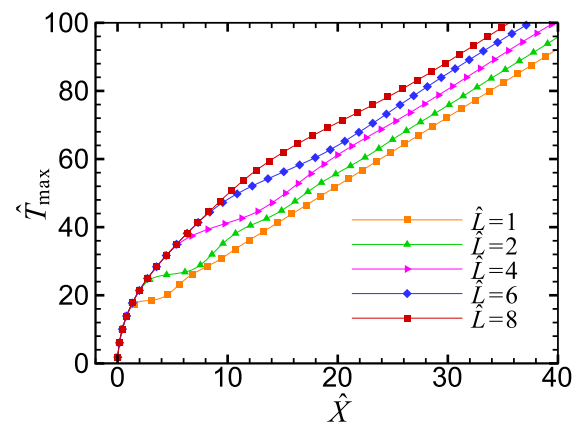

(b)

Figure 8: Effect of plug length on the heat transfer in plug flow: (a) Nusselt number; (b) the maximum fluid temperature. The Peclet number is $P e=100$.
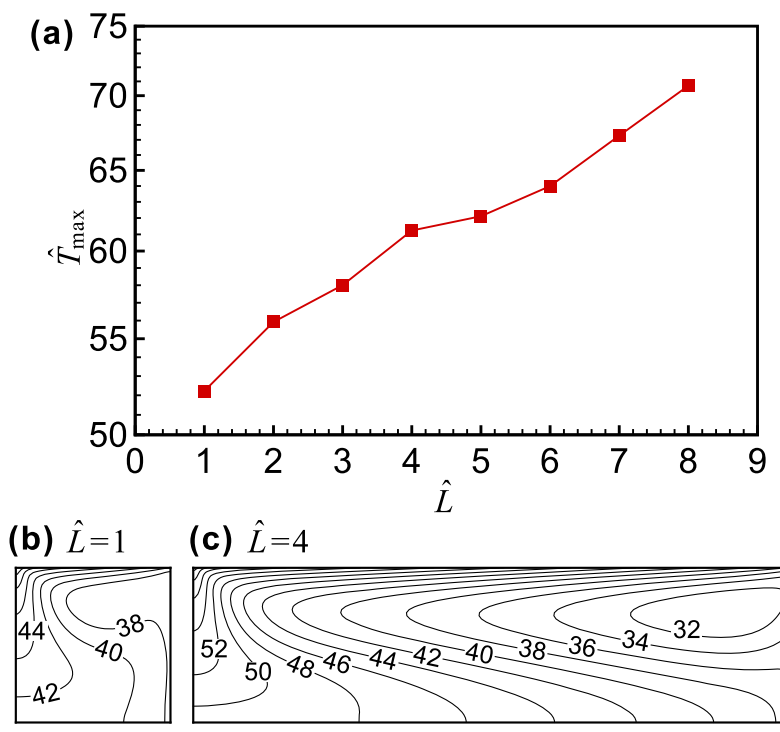

(c) $\hat{L}=4$

(d) $\hat{L}=8$
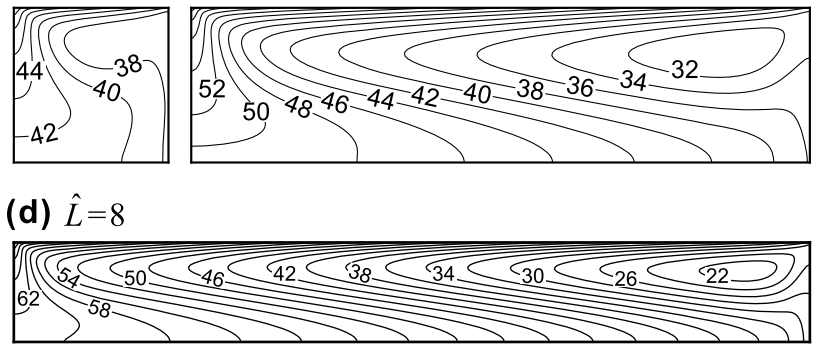

Figure 9: Maximum fluid temperature $\hat{T}_{\max }$ at $\hat{X}=20$ at different plug lengths. (a) Variation of $\hat{T}_{\max }$ with $\hat{L}$; (b)-(d) Temperature contours at different plug lengths $(\hat{L}=$ $1,4,8)$. 


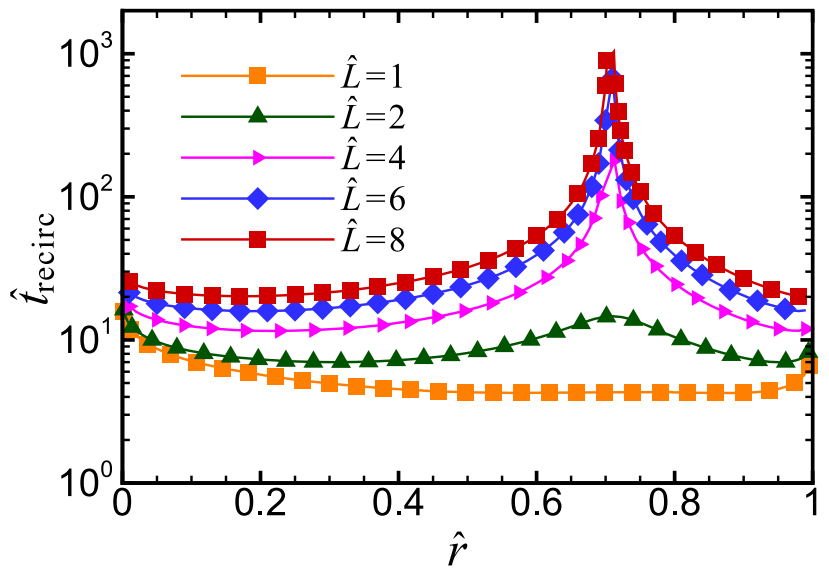

Figure 10: Recirculating period $\hat{t}_{\text {recirc }}$ versus the radial position of fluid particles for plugs of different lengths.

heat transfer are identified. The heat transfer process is evaluated through the Nusselt number and the maximum fluid temperature. The results show that higher Peclet numbers and shorter plug lengths result in higher Nusselt number and lower maximum fluid temperature. In the designing of microchannel heat exchangers, with a constant-surface-heat-flux boundary condition, the plug length and the Peclet number should optimized by considering both the maximum fluid temperature and the flow resistance.

\section{Acknowledgements}

The authors gratefully acknowledge research support from the Singapore Ministry of Education Academic Research Fund Tier 2 research Grant MOE2011-T2-1-036. 


\section{References}

Abbassi, H., Turki, S., Ben Nasrallah, S., 2001. Numerical investigation of forced convection in a plane channel with a built-in triangular prism. International Journal of Thermal Sciences 40, 649-658.

Angeli, P., Gavriilidis, A., 2008. Hydrodynamics of taylor flow in small channels: A review. Proceedings of the Institution of Mechanical Engineers Part C-Journal of Mechanical Engineering Science 222, 737-751.

Che, Z., Wong, T.N., Nguyen, N.T., 2011. An analytical model for plug flow in microcapillaries with circular cross section. International Journal of Heat and Fluid Flow 32, 1005-1013.

Che, Z., Wong, T.N., Nguyen, N.T., 2012. Heat transfer enhancement by recirculating flow within liquid plugs in microchannels. International Journal of Heat and Mass Transfer 55, 1947-1956.

Chen, Y., Cheng, P., 2005. Condensation of steam in silicon microchannels. International Communications in Heat and Mass Transfer 32, 175-183.

Chen, Y., Kulenovic, R., Mertz, R., 2009. Numerical study on the formation of taylor bubbles in capillary tubes. International Journal of Thermal Sciences 48, 234-242.

Ebadian, M.A., Lin, C.X., 2011. A review of high-heat-flux heat removal technologies. Journal of Heat Transfer 133, 110801.

Fujioka, H., Grotberg, J.B., 2004. Steady propagation of a liquid plug 
in a two-dimensional channel. Journal of Biomechanical EngineeringTransactions of the ASME 126, 567-577.

Günther, A., Jhunjhunwala, M., Thalmann, M., Schmidt, M.A., Jensen, K.F., 2005. Micromixing of miscible liquids in segmented gas-liquid flow. Langmuir 21, 1547-1555.

Handique, K., Burns, M.A., 2001. Mathematical modeling of drop mixing in a slit-type microchannel. Journal of Micromechanics and Microengineering $11,548-554$.

Incropera, F.P., DeWitt, D.P., 2002. Fundamentals of Heat and Mass Transfer. John Wiley \& Sons, New York. 5th edition.

Jambunathan, K., Lai, E., Moss, M.A., Button, B.L., 1992. A review of heat transfer data for single circular jet impingement. International Journal of Heat and Fluid Flow 13, 106-115.

Kawahara, A., Chung, P.Y., Kawaji, M., 2002. Investigation of twophase flow pattern, void fraction and pressure drop in a microchannel. International Journal of Multiphase Flow 28, 1411-1435.

Kim, B.J., Liu, Y.Z., Sung, H.J., 2004. Micro piv measurement of two-fluid flow with different refractive indices. Measurement Science \& Technology 15, 1097-1103.

Kim, J., 2007. Spray cooling heat transfer: The state of the art. International Journal of Heat and Fluid Flow 28, 753-767. 
Launay, S., Sartre, V., Bonjour, J., 2007. Parametric analysis of loop heat pipe operation: a literature review. International Journal of Thermal Sciences 46, 621-636.

Lin, L., Ponnappan, R., 2003. Heat transfer characteristics of spray cooling in a closed loop. International Journal of Heat and Mass Transfer 46, $3737-3746$.

Liu, F., Wang, L., 2009. Analysis on multiplicity and stability of convective heat transfer in tightly curved rectangular ducts. International Journal of Heat and Mass Transfer 52, 5849-5866.

Meis, M., Varas, F., Velzquez, A., Vega, J.M., 2010. Heat transfer enhancement in micro-channels caused by vortex promoters. International Journal of Heat and Mass Transfer 53, 29-40.

Morini, G.L., 2004. Single-phase convective heat transfer in microchannels: A review of experimental results. International Journal of Thermal Sciences $43,631-651$.

Muradoglu, M., Gnther, A., Stone, H.A., 2007. A computational study of axial dispersion in segmented gas-liquid flow. Physics of Fluids 19, 072109.

Muzychka, Y.S., Walsh, E., Walsh, P., 2010. Simple models for laminar thermally developing slug flow in noncircular ducts and channels. Journal of Heat Transfer 132, 111702.

Naphon, P., Wongwises, S., 2006. A review of flow and heat transfer characteristics in curved tubes. Renewable and Sustainable Energy Reviews 10, 463-490. 
Oishi, M., Kinoshita, H., Fujii, T., Oshima, M., 2009. Confocal micro-piv measurement of droplet formation in a t-shaped micro-junction. Journal of Physics: Conference Series 147, 012061.

Osher, S., Fedkiw, R.P., 2001. Level set methods: an overview and some recent results. Journal of Computational Physics 169, 463-502.

Palm, B., 2001. Heat transfer in microchannels. Microscale Thermophysical Engineering 5, 155-175.

Patankar, S.V., 1980. Numerical Heat Transfer and Fluid Flow. Series in computational methods in mechanics and thermal sciences, Hemisphere Pub. Corp., Washington.

Peng, X.F., Wang, B.X., 1993. Forced convection and flow boiling heat transfer for liquid flowing through microchannels. International Journal of Heat and Mass Transfer 36, 3421-3427.

Scardovelli, R., Zaleski, S., 1999. Direct numerical simulation of free-surface and interfacial flow. Annual Review of Fluid Mechanics 31, 567-603.

Song, H., Bringer, M.R., Tice, J.D., Gerdts, C.J., Ismagilov, R.F., 2003a. Experimental test of scaling of mixing by chaotic advection in droplets moving through microfluidic channels. Applied Physics Letters 83, 46644666.

Song, H., Tice, J.D., Ismagilov, R.F., 2003b. A microfluidic system for controlling reaction networks in time. Angewandte Chemie - International Edition 42, 768-772. 
Taha, T., Cui, Z.F., 2004. Hydrodynamics of slug flow inside capillaries. Chemical Engineering Science 59, 1181-1190.

Thome, J.R., 2004. Boiling in microchannels: A review of experiment and theory. International Journal of Heat and Fluid Flow 25, 128-139.

Triplett, K.A., Ghiaasiaan, S.M., Abdel-Khalik, S.I., Sadowski, D.L., 1999. Gas-liquid two-phase flow in microchannels part i: Two-phase flow patterns. International Journal of Multiphase Flow 25, 377-394.

Turki, S., Abbassi, H., Ben Nasrallah, S., 2003. Two-dimensional laminar fluid flow and heat transfer in a channel with a built-in heated square cylinder. International Journal of Thermal Sciences 42, 1105-1113.

Vasiliev, L.L., 2005. Heat pipes in modern heat exchangers. Applied Thermal Engineering 25, 1-19.

Walsh, P.A., Walsh, E.J., Muzychka, Y.S., 2010. Heat transfer model for gas-liquid slug flows under constant flux. International Journal of Heat and Mass Transfer 53, 3193-3201.

Yu, Z., Hemminger, O., Fan, L.S., 2007. Experiment and lattice boltzmann simulation of two-phase gas-liquid flows in microchannels. Chemical Engineering Science 62, 7172-7183.

Zhang, J., 2010. Lattice boltzmann method for microfluidics: models and applications. Microfluidics and Nanofluidics 10, 1-28. 